\title{
Caligus rogercresseyi acetylcholinesterase types and variants: a potential marker for organophosphate resistance
}

\author{
Celia Agusti-Ridaura ${ }^{* *}$ (D, Michael Dondrup ${ }^{2}$, Tor E. Horsberg ${ }^{1}$, Jong S. Leong ${ }^{3}$, Ben F. Koop³ Sandra Bravo ${ }^{4}$,
} Julio Mendoza ${ }^{5}$ and Kiranpreet Kaur ${ }^{1}$

\begin{abstract}
Background: Control of the sea louse Caligus rogercresseyi in the Chilean salmonid industry is reliant on chemical treatments. Azamethiphos was introduced in 2013, although other organophosphates were previously used. In 2014, reduced sensitivity to azamethiphos was detected in the Los Lagos Region using bioassays. The main target of organophosphates is the enzyme acetylcholinesterase (AChE). Mutations in the AChE gene are the main cause of organophosphate resistance in arthropods, including other sea lice. In the present study, we aimed to characterize C. rogercresseyi AChE(s) gene(s) and to study the association between AChE variants and azamethiphos resistance in this sea louse species.

Methods: Samples of adult male and female C. rogercresseyi were collected in the Los Lagos Region in 2014. Twenty-four hour exposure bioassays with azamethiphos were performed to select sensitive and resistant lice. The full-length cDNA coding sequences encoding for two AChEs in C. rogercresseyi were molecularly characterized. One of the AChE genes was screened by direct sequencing in the azamethiphos-selected lice to search for variants. An additional louse sampling was performed before and after an azamethiphos treatment in the field in 2017 to validate the findings.

Results: The molecular analysis revealed two putative AChEs in C. rogercresseyi. In silico analysis and 3D modelling of the protein sequences identified both of them as invertebrate AChE type 1; they were named C. rogercresseyi AChE1a and 1b. AChE1a had the characteristics of the main synaptic AChE, while AChE1b lacked some of the important amino acids of a typical AChE. A missense change found in the main synaptic AChE (1a), F318FN (F290 in Torpedo californica), was associated with survival of $C$. rogercresseyi at high azamethiphos concentrations (bioassays and field treatment). The amino acid change was located in the acyl pocket of the active-site gorge of the protein.

Conclusions: The present study demonstrates the presence of two types of AChE1 genes in C. rogercresseyi. Although enzymatic assays are needed, AChE1a is most probably the main synaptic AChE. The function of AChE1b is unknown, but evidence points to a scavenger role. The AChE1a FN318 variant is most probably involved in organophosphate resistance, and can be a good marker for resistance monitoring.
\end{abstract}

Keywords: Caligus rogercresseyi, Acetylcholinesterase, Organophosphate, Azamethiphos, Resistance, Variants, Mutation

\footnotetext{
* Correspondence: celia.agusti@nmbu.no; agusti.celia@gmail.com

${ }^{1}$ Faculty of Veterinary Medicine, Norwegian University of Life Sciences, Sea

Lice Research Centre, Postboks 369 Sentrum, Oslo NO-0102, Norway

Full list of author information is available at the end of the article
}

(c) The Author(s). 2018 Open Access This article is distributed under the terms of the Creative Commons Attribution 4.0 International License (http://creativecommons.org/licenses/by/4.0/), which permits unrestricted use, distribution, and reproduction in any medium, provided you give appropriate credit to the original author(s) and the source, provide a link to the Creative Commons license, and indicate if changes were made. The Creative Commons Public Domain Dedication waiver (http://creativecommons.org/publicdomain/zero/1.0/) applies to the data made available in this article, unless otherwise stated. 


\section{Background}

The sea louse Caligus rogercresseyi (Copepoda, Caligidae) is the most harmful parasite affecting the salmon farming industry in Chile [1-3]. Heavily infested fish show physiological changes due to stress, lice-induced skin lesions, weight loss and increased susceptibility towards other infectious diseases [4]. In addition to the economic loss due to the damage inflicted to fish, salmon producers must face the additional cost of anti-lice treatments. Caligus rogercresseyi has a wide host range, parasitizing both wild and farmed fish (reviewed in [5]). Its direct life-cycle facilitates the transmission between hosts, especially in densely reared fish [5].

Control of C. rogercresseyi infestations in Chilean fish farms depends mainly on the use of anti-lice chemicals (reviewed in $[6,7])$. The organophosphates metrifonate and dichlorvos (applied by bath) were used during the period 1981-2000 [8]. Between 2000 and 2007, the only anti-lice chemical authorized in Chile was the avermectin emamectin benzoate $[9,10]$. Reduction in treatment efficacy by emamectin benzoate was first detected in 2006-2007 [911]. Pyrethroids were introduced (deltamethrin in 2007 and cypermethrin in 2010) to cope with the resistance problem of C. rogercresseyi towards emamectin benzoate. Pyrethroids became the main anti-lice chemical agents in use until 2013, when treatment failures due to pyrethroid-resistant parasites were reported [12]. In order to control $C$. rogercresseyi loads in salmonid farms, the organophosphate azamethiphos was introduced in 2013 [7]. Since then, azamethiphos is the most utilized anti-lice chemical in Chilean salmonid farming [13].

Sea lice resistance towards the main anti-lice chemicals has been recorded in most salmonid producing countries. Organophosphate-resistant salmon lice, Lepeophtheirus salmonis, have been found in Scotland, Norway and Ireland [14-17]. Reduced sensitivity of C. rogercresseyi towards azamethiphos has been reported recently in some salmonid production areas in Chile [7, 18]. Agusti et al. [7] showed that azamethiphos treatment efficacy for adult C. rogercresseyi ranged between 92 and 100\% in four farms located in Los Lagos and Aysén Regions (commonly known as Regions X and XI, respectively). However, when lice sensitivity to azamethiphos was tested in Los Lagos Region by the use of bioassays, Agusti et al. [7] reported $\mathrm{EC}_{50}$ values (the concentration immobilizing $50 \%$ of the parasites) between eight and fifteen times higher than the C. rogercresseyi putative naïve level (sensitivity of lice never exposed to an anti-louse chemical). Marín et al. [18] also observed reduced sensitivity of $C$. rogercresseyi to azamethiphos in several farms in Los Lagos and Aysén Regions, and found that lice from Los Lagos Region tended to be less sensitive to azamethiphos than those from Aysén Region. Agusti et al. [7] suggested the previous (1981-2001) and present (2013-2016) use of organophosphates as the possible cause for the reduced sensitivity observed in Los Lagos Region.

Due to the incipient resistance development in C. rogercresseyi to azamethiphos, an accurate time-space monitoring of the sensitivity level to this chemical is highly necessary. Currently, bioassays are the only available method for measuring C. rogercresseyi sensitivity in Chile $[7,12,18]$. In these assays, live sea lice are exposed to a range of chemical concentrations for a given time. Following the exposure, the number of dead or immobilized parasites is registered [19]. However, bioassays are laborious to conduct and subject to several potential methodological and interpretation errors (discussed in [20, 21]). Molecular methods have demonstrated to be a powerful tool for monitoring the sensitivity of sea lice to chemicals $[22,23]$. However, it is important to elucidate the molecular mechanisms behind the resistance for developing such a tool.

Mutations in the gene coding for the enzyme acetylcholinesterase (AChE) have been reported as the main cause of resistance towards organophosphates in arthropods, followed by metabolic and behavioural mechanisms (discussed in [21, 24-28]). AChE is present in the cholinergic synapses of the nervous system of arthropods and in the neuromuscular junctions of vertebrates [29]. It hydrolyses the neurotransmitter acetylcholine, assuring the correct functioning of neurotransmission. Organophosphates inhibit AChE, causing the accumulation of acetylcholine in the synapses (reviewed in $[24,25,28,30]$ ). This results in overstimulation of nerves and muscles, leading to paralysis and death of the parasite. Kaur et al. [21, 22] found that a single amino acid mutation (F362Y, corresponding to Phe331 in Torpedo californica AChE) in the active-site gorge of the synaptic AChE was the major factor responsible for organophosphate resistance in $L$. salmonis. Based on those findings, a rapid and reliable assay was developed for screening the mutation in field samples, providing a valuable tool for salmon louse resistance monitoring [21, 22].

The main aim of the present study was to identify and characterize the AChEs present in adult $C$. rogercresseyi collected in Chilean salmonid farms. In order to explore the possible causes for the loss of sensitivity to azamethiphos in some louse populations, the gene coding for the synaptic $\mathrm{AChE}$ enzyme (C. rogercresseyi AChE1a) was screened for variants in sensitive and resistant lice.

\section{Methods}

\section{Sea lice sampling and selection}

Caligus rogercresseyi individuals were collected at twotime points: during March 2014 and June 2017. Three sampling sites were chosen based on the availability of lice in farms in Los Lagos Region and the suspected different lice sensitivities to azamethiphos [7, 18]. The number of treatments applied in each farm and the 
treatment efficacies were used as an indicator for farm selection, since low lice sensitivity seems to be related with high number of treatments and low treatment efficacies $[7,23]$. The farms selected were located in the north and middle part of the interior sea in Los Lagos Region (Fig. 1). During the year before the lice were collected, Farm 1 conducted two azamethiphos treatments and Farm 2 seven treatments before March 2014; Farm 3 conducted four azamethiphos treatments before lice sampling in June 2017. All farms were stocked with a single year-class of fish. Farms 1 and 3 reared Atlantic salmon and Farm 2 reared rainbow trout. Fish were sampled using a dip net and anesthetized for louse collection.

Bioassays were conducted on lice collected in Farms 1 and 2 during March 2014 in order to assess the actual lice sensitivity to azamethiphos and for a proper selection of sensitive versus resistant lice. Lice used for bioassays were all adult males and females, but differences in the maturation level of the genital segment were observed. Bioassays were carried out as described by Agusti et al. [7], Helgesen et al. [12] and Helgesen \& Horsberg [19]. Caligus rogercresseyi were gently removed from the anesthetized fish using forceps. They were kept alive at low temperature in containers with seawater supplied with aeration until the start of the bioassay. Sea lice were exposed to azamethiphos for $24 \mathrm{~h}$ at $12{ }^{\circ} \mathrm{C}$ with constant aeration, and the results were recorded immediately following the exposure time. Two types of bioassays were designed depending on the number of azamethiphos dilutions used and the purpose of the bioassay. For sensitivity assessment (and lice selection), four to five azamethiphos dilutions were prepared in filtered (100 $\mu \mathrm{m}$ filter) seawater, ranging from 0.2 to $2 \mathrm{ppb}(\mu \mathrm{g}$ $\left.\mathrm{l}^{-1}\right)$. For lice selection only, two azamethiphos dilutions were used: 0.4 and $2 \mathrm{ppb}$. Lice immobilized (unable to attach to the walls of the containers or swim actively) at $0.4 \mathrm{ppb}$ or below, were considered sensitive, while lice unaffected at $2 \mathrm{ppb}$ were considered resistant $[7,19,22,23]$. Immediately following the 24 $\mathrm{h}$ exposure, lice were fixed in RNAlater (Sigma-Merck KGaA, Darmstadt, Germany; item number R0901) and kept at $-80{ }^{\circ} \mathrm{C}$. Control groups without azamethiphos were prepared to determine the general performance of the lice. Bioassays were initiated within 2-6 h of sampling the first louse. Data from four to five dose bioassays were modelled using probit modelling in JMP software, and $\mathrm{EC}_{50}$ values with $95 \%$ confidence intervals were calculated separately for males and females.

Fifty-seven additional lice were collected in June 2017 before and after an azamethiphos treatment event in Farm 3. Pre-treatment louse sampling was performed one day before the treatment. Thirty adult lice (12 males and 18 females) were collected from five fish during the pre-treatment sampling. The azamethiphos treatment was applied by bath at a concentration of $0.1 \mathrm{~g} / \mathrm{m}^{3}$ for $50 \mathrm{~min}$. The post-treatment sampling occurred three days after treatment, lice being collected from the same cage as the pre-treatment sampling. Twenty-eight adult lice (20 males and 8 females) were recovered after treatment from 20 fish. Lice were gently removed from the anesthetized fish using forceps and were transferred directly to RNAlater ${ }^{\ominus}$ for fixation and preservation.

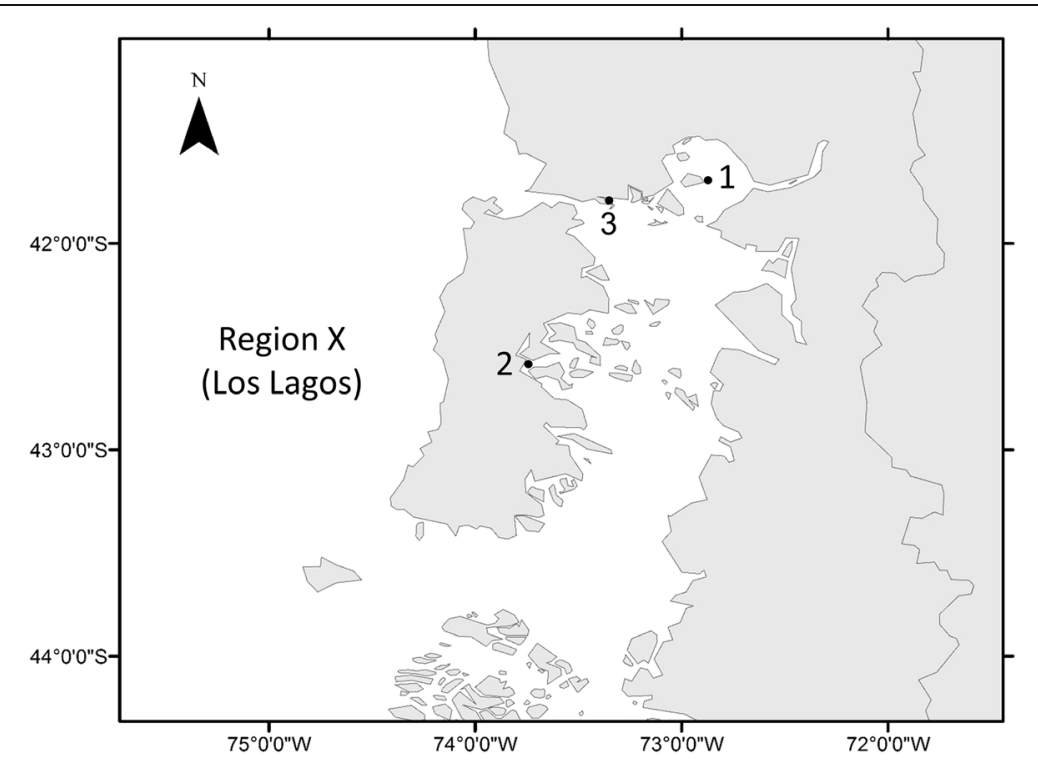

Fig. 1 Farm locations where Caligus rogercresseyi were collected in March 2014 (Farms 1 and 2) and in June 2017 (Farm 3). Map displays the interior sea in Los Lagos Region (Region X) 
RNA extraction and CDNA synthesis

Total RNA was extracted from the whole body (lice from Farms 1 and 2; bioassays) or only from the cephalotorax (lice from Farm 3; pre-post azamethiphos treatment) of adult $C$. rogercresseyi females and males. A Trizol protocol combined with the RNeasy Mini kit for animal tissues (Qiagen, Hilden, Germany) was used for the extraction. Lice tissues were homogenized in $1 \mathrm{ml}$ Trizol using TissueLyser MM 301 (Qiagen Retsch, Hilden, Germany) and one stainless steel bead of $5 \mathrm{~mm}$ diameter (Qiagen). After mixing with $0.2 \mathrm{ml}$ chloroform and a centrifugation step, the aqueous phase was transferred to a new vial and mixed with one volume of $70 \%$ ethanol. Total RNA was then isolated with RNeasy spin columns following the manufacturer's protocol. The RNA was quantified with an ND-100 Spectrophotometer (Thermo Fisher Scientific, Waltham, Massachusetts, USA) and the quality was checked with a 2100 Bioanalyzer instrument (Agilent Technologies, Santa Clara, California, USA) and an Agilent RNA 6000 Nano kit. First strand cDNA was synthesized with the RevertAid H Minus first strand cDNA synthesis kit (Thermo Fisher Scientific) using oligo $(\mathrm{dT})_{18}$ primers.

\section{Identification and characterization of $C$. rogercresseyi AChEs}

In order to identify $C$. rogercresseyi $\mathrm{AChE}$ sequences, a search against the C. rogercresseyi transcriptome [31] was conducted using the two types of AChEs in L. salmonis (ace1a and $a c e 1 b$ ) as queries. The search was performed using blastn optimized for discontiguous megablast [32]. To verify and properly characterize the $C$. rogercresseyi sequences obtained, the cDNA sequences were used for searching against the $C$. rogercresseyi Whole-Genome Shotgun Contigs (WGS) [33] (blastn, megablast, NCBI [32]). The cDNA and genomic sequences were assembled using ContigExpress software (Vector NTI Advance 11.0, Invitrogen Corp. 2008). The sequences obtained were amplified and analyzed on a subset of lice enrolled in the bioassay analysis $(n=10)$. The PCR cycling conditions were as follows: $98^{\circ} \mathrm{C}$ for 2 min followed by 35 cycles of amplification at $98^{\circ} \mathrm{C}$ for $10 \mathrm{~s}, 62^{\circ} \mathrm{C}$ or $67^{\circ} \mathrm{C}$ for $15 \mathrm{~s} \mathrm{(see}$ Additional file 1 for primer annealing temperature), $72{ }^{\circ} \mathrm{C}$ for $2 \mathrm{~min}$ and a final extension step at $72^{\circ} \mathrm{C}$ for $5 \mathrm{~min}$. The primers selected were located in the putative untranslated $5^{\prime}$ and $3^{\prime}$ regions (UTRs) and are listed in Additional file 1 . The Phusion ${ }^{\circ}$ High-Fidelity DNA Polymerase (New England BioLabs, Ipswich, Massachusetts, USA) was utilized. Amplicons were subjected to direct sequencing using several internal primers (Additional file 1) (GATC Biotech, Constance, Germany; Sanger sequencing service, Supremerun sequencing).

In silico analysis of the deduced C. rogercresseyi $\mathrm{AChE}$ protein sequences were performed as follows. Database searches of the sequences were conducted against several databases in order to verify the identity of the proteins: GenBank (non-redundant protein sequences - nr, BlastP), UniProtKB (BlastP; default settings) and InterProScan sequence search (default settings). In addition, the deduced proteins were compared to previously published $\mathrm{AChE}$ protein sequences from another 19 species, using the ClustalO program [34] with default settings to obtain a multiple sequence alignment (MSA). The following online modelling platforms were used for predicting the presence of transmembrane helices in C. rogercresseyi AChEs: SOSUI [35, 36], TMHMM Server v.2.0 [37-39] and DAS [40, 41].

A phylogenetic analysis of the putative $C$. rogercresseyi AChEs was performed. Several cholinesterase (ChE) types from vertebrates and invertebrates were included. Full-length ChE sequences were found by: (i) BlastP searches of the GenBank-nr and UniprotKB databases using the $C$. rogercresseyi $\mathrm{AChE}$ sequences identified in this study as queries; and (ii) searching for complete sequences in the UniprotKB database, using keywords as queries ("acetylcholinesterase", "cholinesterase", "ace", "AChE", etc.). MSA was generated with MUSCLE [42] with default parameters. MSA was manually inspected and edited in Jalview [43] removing regions of low coverage, low alignment quality, and a complete sequence introducing large gaps (Gallus gallus AChE). Phylogenetic trees were constructed using MrBayes v.3.2.6 x64 [44] using 8 parallel chains over a total of 5 million generations; rates were approximated by a $\Gamma$-distribution with 4 categories, allowing for invariant sites. Markov Chain Monte Carlo (MCMC) analysis was run in mixed mode [45] to allow MrBayes to determine the optimal substitution matrix, resulting in a WAG $+\mathrm{I}+\Gamma$ model. In addition, the MSA was submitted to ProtTest server [46] to determine the optimal substitution model and MrBayes was run again using the best-fit $\mathrm{LG}+\mathrm{I}+\Gamma$ model. The vertebrate taxa were used as out-group to root the trees.

To further explore the identity of $C$. rogercresseyi $\mathrm{AChE}$ sequences, another phylogenetic analysis was performed with the same parameters. The analysis included sequences of several invertebrate and vertebrate carboxylesterases, esterases and other cholinesterases available in the UniprotKB database.

\section{D modelling of $C$. rogercresseyi AChEs and docking analysis}

The three-dimensional (3D) structure of the C. rogercresseyi AChEs was modelled using SWISS MODEL in the automated mode [47-49]. For a better search of templates, a number of amino acids at each end of the C. rogercresseyi AChEs sequences were truncated following a similar pattern as in Drosophila melanogaster and Homo sapiens AChE [Protein Data Bank (PDB) codes 1qo9.1 and 4ey4.1, respectively]. In short, the AChEs sequences were aligned using ClustalO program 
with default settings. The amino acids at the beginning and/or the end of the C. rogercresseyi AChEs that did not align to the other sequences were truncated. QMEAN and GMQE (global model quality estimation) scores were used as indicators of the quality of the models [47]. To analyze the fit between the selected templates and the predicted $C$. rogercresseyi AChEs structures, the root mean square (RMS) values for carbon alpha $(\mathrm{C} \alpha)$ were calculated using the Swiss PDB viewer v.4.1.0 [50]. The values were calculated for the whole protein and for the ten amino acids important for the function of the protein (the anionic choline binding site, the catalytic triad, the acyl pocket residues and the oxyanion hole). The numbering of amino acids is by convention from the T. californica AChE sequence. The 3D models were visualized using UCSF Chimera v.1.10.2. software [51]. The distances between amino acids in the active-site gorge were calculated using the same software. The volume of the active-site gorge of the proteins was calculated using the software CASTp [52, 53].

Acetylcholine and two organophosphates, azamethiphos and dichlorvos, were docked to the $C$. rogercresseyi AChEs $3 \mathrm{D}$ models using the online molecular docking server $[54,55]$. The whole active-site gorge of the proteins, from the mouth to the bottom of the gorge, was selected for performing the analysis. The results were examined for the likely binding sites, frequency of binding to these sites, free binding energy and the estimated inhibition constant (Ki, the concentration required to produce half-maximum inhibition).

\section{Screening of $C$. rogercresseyi ace $1 a$ for variants}

The full cDNA coding sequence of the putative synaptic AChE (1a) was amplified to look for variants, using gene specific primers (Additional file 1, "Cr_ace1a"). The sequence was analyzed in five azamethiphos sensitive, three with reduced sensitivity and two resistant adult $C$. rogercresseyi selected with bioassays (Farms 1 and 2). PCR reactions were performed using Phusion ${ }^{\circ}$ High-Fidelity DNA Polymerase; PCR conditions and direct sequencing were performed as stated in the previous section "Methods. Identification and characterization of $C$. rogercresseyi AChEs".

For rapid screening of the AChE1a variants in the 57 additional lice collected before and after the azamethiphos field treatment (Farm 3, June 2017), a 484 bp fragment of the ace1a gene containing an important missense change was amplified. Two fragment-specific primers were designed (Additional file 1, named HS4F and HS6R). PCR reactions were performed using Phusion ${ }^{\circ}$ High-Fidelity DNA Polymerase under the following conditions: $98^{\circ} \mathrm{C}$ for 2 min followed by 35 cycles of amplification at $98^{\circ} \mathrm{C}$ for $10 \mathrm{~s}, 62{ }^{\circ} \mathrm{C}$ for $20 \mathrm{~s}, 72{ }^{\circ} \mathrm{C}$ for $30 \mathrm{~s}$ and a final extension step at $72{ }^{\circ} \mathrm{C}$ for $5 \mathrm{~min}$. Amplicons were subjected to direct sequencing (GATC Biotech; Sanger sequencing service, Supremerun sequencing).

\section{Results \\ Sequence analysis and characterization of C. rogercresseyi AChEs}

Two partial cDNA AChE sequences were identified in $C$. rogercresseyi transcriptome ([31] GenBank: GAZX01027370.1 and GAZX01029466.1). The search of these sequences against the $C$. rogercresseyi WGS (female and male) resulted in several contigs (see Additional file 2). The cDNA sequence of GAZX01027370.1, identified in the C. rogercresseyi transcriptome as a putative AChE, was confirmed and characterized in the lice collected in the present study $(n=10)$. It has an open reading frame (ORF) of $1941 \mathrm{bp}$, encoding a deduced protein of 646 amino acids. In silico analysis of this deduced protein identified it as an AChE with transmembrane segment. The domains and signatures identified were: cholinesterase, alpha/beta hydrolase fold, carboxylesterase type B-active site, cytoplasmic region, non-cytoplasmic region, transmembrane region and several signal peptides. The amino acid alignment of this $C$. rogercresseyi putative AChE with $19 \mathrm{AChE}$ sequences from other species (Crustacea, Insecta, Nematoda, Arachnida and Vertebrata) revealed that it has the characteristic features of the main synaptic AChE (Fig. 2 and Additional file 3) [56]. These features include: the anionic choline binding site (W84, numbering corresponds to T. californica $\mathrm{AChE}$ ), the three residues of the catalytic triad (S200, E327 and H440), the characteristic FGESAG motif surrounding the active serine, the acyl pocket residues (W233, F290 and F331), the oxyanion hole (G118, G119 and A201) and six cysteines involved in the three conserved disulphide bonds (C67-C94, C254-C265, C402-C521). Three online modeling platforms (SOSUI, TMHMM Server v.2.0 and DAS; see Methods for details) identified a hydrophobic C-terminal peptide in this $C$. rogercresseyi $\mathrm{AChE}$ as a transmembrane helix, between the amino acids 604 and 626 (C. rogercresseyi AChE numbering) (Fig. 3). The alignment of C. rogercresseyi AChE with L. salmonis AChE1a revealed a high identity between them (85.3\%), while the identity percentage with $L$. salmonis AChE1b was $77.2 \%$. This alignment also identified the putative $C$. rogercresseyi AChE hydrophobic peptide in a similar position as in $L$. salmonis AChE1a (Fig. 3), and the most probable cleavage site of the C. rogercresseyi AChE hydrophobic peptide in the cysteine residue C595 (also C595 in L. salmonis) (Fig. 3). Based on these observations, we concluded that this $C$. rogercresseyi AChE could be the main synaptic enzyme.

When the other cDNA sequence, GAZX01029466.1 (preliminarily identified as another AChE in the $C$. 


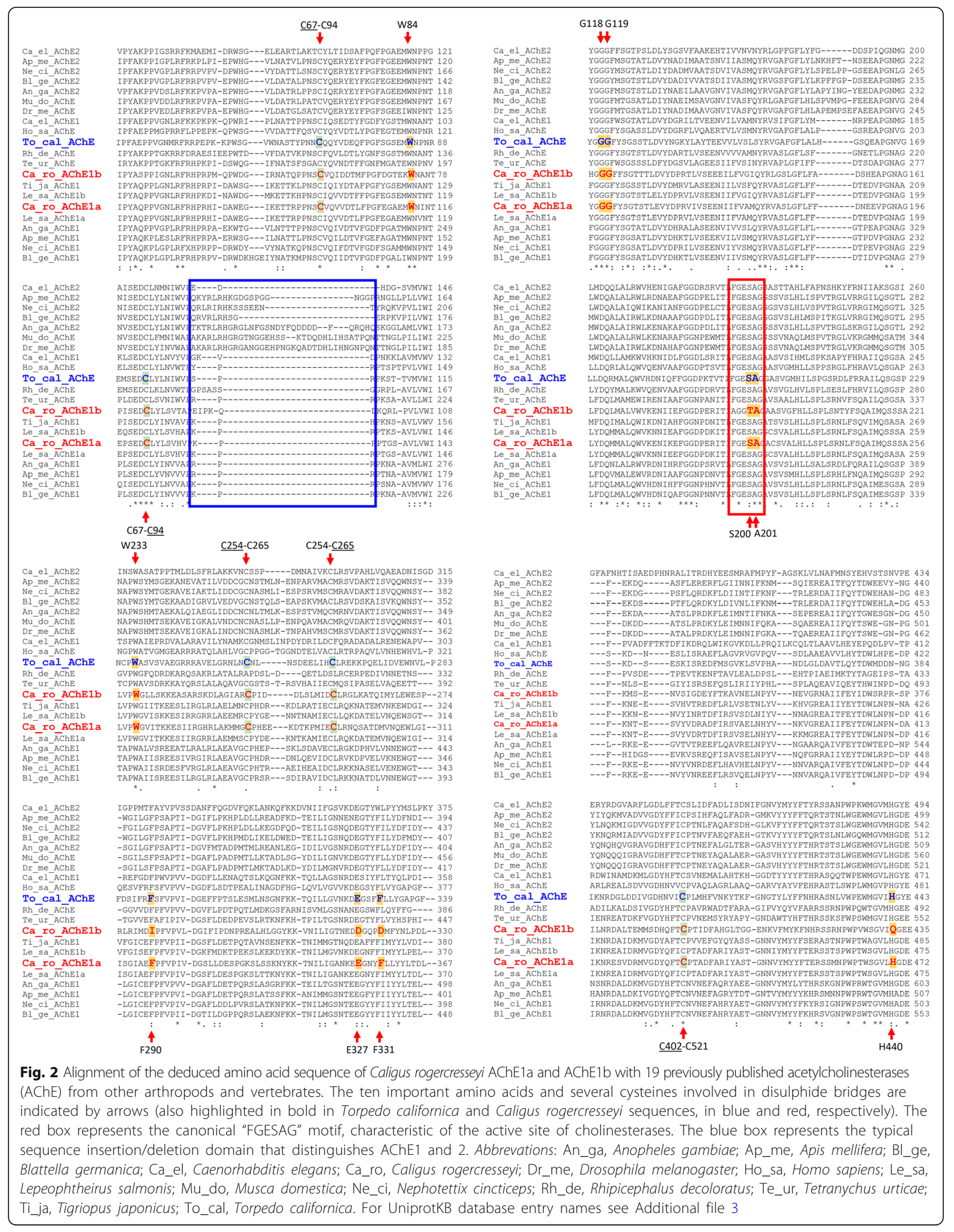




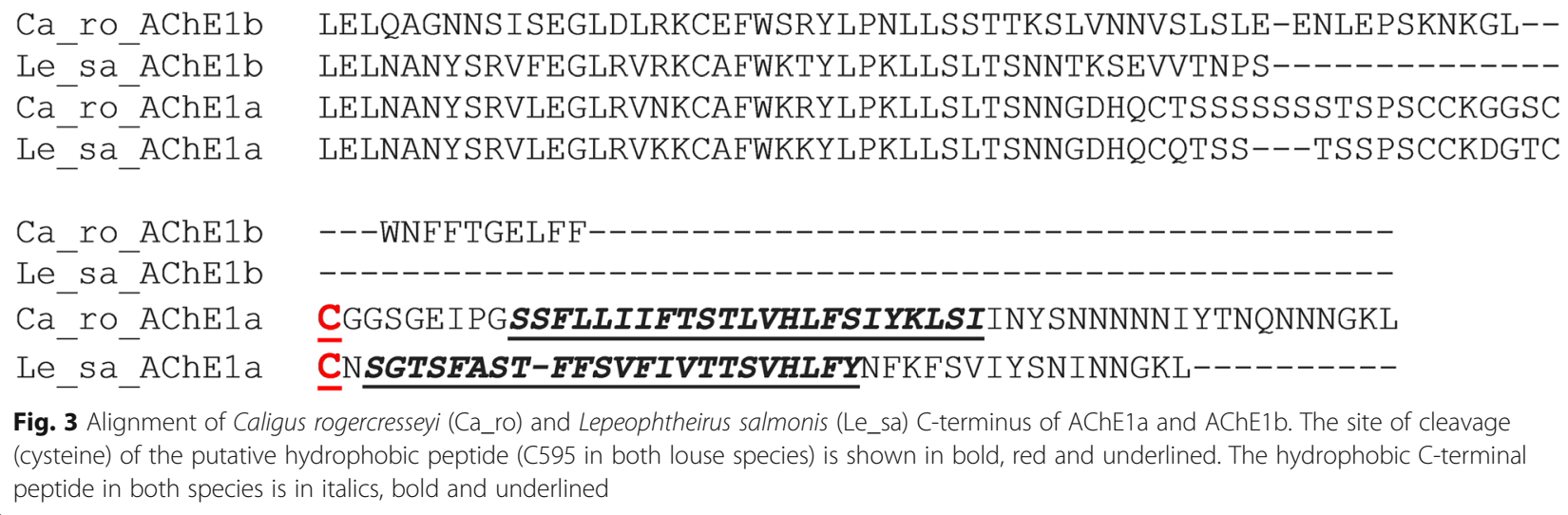

Fig. 3 Alignment of Caligus rogercresseyi (Ca_ro) and Lepeophtheirus salmonis (Le_sa) C-terminus of AChE1a and AChE1b. The site of cleavage (cysteine) of the putative hydrophobic peptide (C595 in both louse species) is shown in bold, red and underlined. The hydrophobic C-terminal peptide in both species is in italics, bold and underlined

rogercresseyi transcriptome), is translated into protein, the putative start codon is located only $22 \mathrm{bp}$ from the beginning of the sequence. We searched in the C. rogercresseyi WGS to obtain a longer 5 ' end fragment of the sequence. The search resulted in five genome contigs overlapping with the cDNA sequence (Additional file 2). The GAZX01029466.1 sequence was assembled with the genome contigs obtained in the blast analysis. The consensus sequence increased the putative $5^{\prime}$ end by 279 additional bp. Primers were then designed in the new putative untranslated $5^{\prime}$ region (UTR) and in the $3^{\prime}$ region (Additional file 1, "Cr_ace1b"). The PCR product obtained using those primers revealed a transcript longer than the consensus sequence obtained from the assembling of GAZX01029466.1 and the genome contigs, since a completely new fragment of 484 bp was found. This fragment comprises 440 bp belonging to the $5^{\prime}$ UTR and $44 \mathrm{bp}$ to the beginning of the coding sequence, containing the "atg" start codon. The new transcript has an ORF of $1689 \mathrm{bp}$, encoding a deduced protein 562 aa long. The new $5^{\prime}$ UTR is at least $776 \mathrm{bp}$ long. The sequence was submitted to the GenBank database under the accession number MF978386. In silico analysis of the deduced protein revealed that it probably was an AChE without transmembrane segment. The domains and signatures identified were: cholinesterase, alpha/beta hydrolase fold and carboxylesterase type B; no cytoplasmic or transmembrane regions were identified. When the putative protein sequence was aligned with 19 $\mathrm{AChE}$ sequences from other species, only some of the features of a typical AChE were identified (Fig. 2): it has the anionic choline binding site (W84), the oxyanion hole (G118, G119 and A201) and six cysteine residues involved in three conserved disulphide bonds (C67-C94, C254C265, C402-C521). However, all three residues of the catalytic triad and two residues of the acyl pocket are substituted by other amino acids, and it lacks the characteristic FGESAG motif. The three online modeling platforms used for predicting the presence of transmembrane helices (SOSUI, TMHMM Server v. 2.0 and DAS), predicted this protein to be soluble, without hydrophobic peptide. In addition, no free cysteine residue (the potential cleavage site of a hydrophobic peptide) is present in the C-terminal of the protein (Fig. 3), as is the case in L. salmonis AChE1b. The most similar proteins identified were L. salmonis AChE1a (54.9\%) and AChE1b (54.9\%). It shares $54 \%$ similarity with the other AChE described above for $C$. rogercresseyi.

Phylogenetic analysis (Fig. 4 and Additional files 3 and 4) showed that the two putative $C$. rogercresseyi $\mathrm{AChE}$ sequences cluster together with the AChE type 1 occurring in crustaceans and insects, being clearly separated from the AChE type 2 group. Based on this finding, the protein deduced from the GAZX01027370.1 sequence will be hereafter referred to as C. rogercresseyi AChE1a, and the protein deduced from the new sequence generated on basis of GAZX01029466.1, as C. rogercresseyi AChE1b. Caligus rogercresseyi AChE1a grouped together with both $L$. salmonis AChEs in the phylogenetic tree. Caligus rogercresseyi $\mathrm{AChE1b}$ appeared in the crustacean AChE type 1 cluster, but with a large genetic distance. The inclusion of several invertebrate and vertebrate carboxylesterases, esterases and other cholinesterases did not change the position of $C$. rogercresseyi $\mathrm{AChE1b}$ in the tree (Additional file 4).

\section{Bioassays and field treatment with azamethiphos}

Bioassays with $24 \mathrm{~h}$ exposure time were performed to determine the lice sensitivity towards azamethiphos, at the population and individual levels. In Farms 1 and 2, one 5/ 6-dose bioassay was performed per farm. The lice sensitivity level to azamethiphos, expressed as $\mathrm{EC}_{50}$ values with 95\% confidence intervals, was $0.48 \mathrm{ppb}(0.09-2.50)$ for males and $0.51 \mathrm{ppb}(0.26-0.99)$ for females in Farm 1, and $1.20 \mathrm{ppb}(1.19-1.22)$ for both males and females in Farm 2. In addition, a 3-dose bioassay for lice selection was conducted in Farm 2. Table 1 summarizes the immobilization data of $C$. rogercresseyi exposed to different azamethiphos concentrations per farm. 


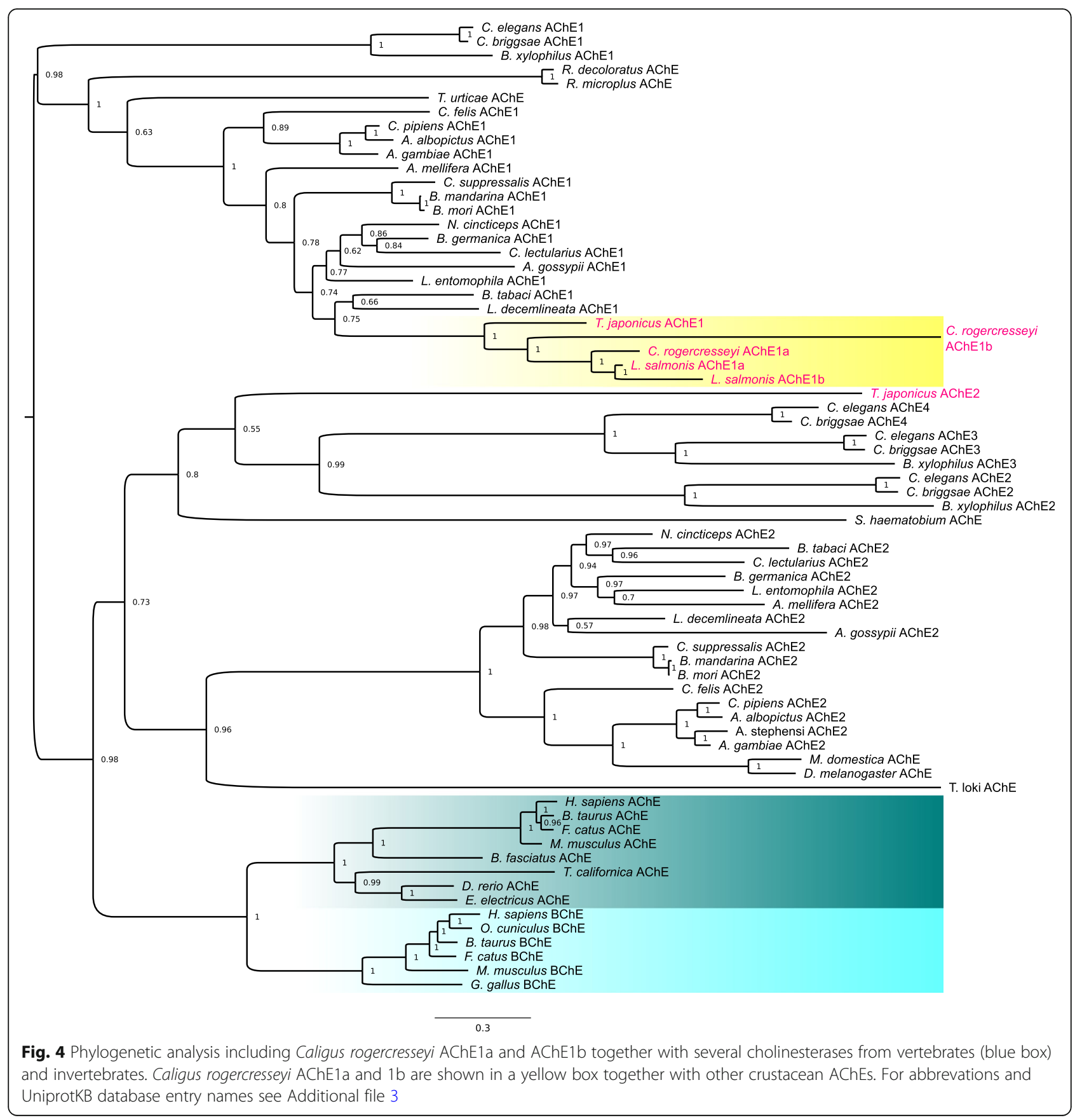

An azamethiphos field treatment in Farm 3 was used for testing the association of an important missense change found in ace1a with C. rogercresseyi sensitivity loss towards azmethiphos. Male and female lice collected before the treatment were adults with the genital segment completely developed ("mature" following the terminology by González \& Carvajal [5]), and some females carried egg strings. Treatment efficacy in the sampling cage was $97 \%$ for adult lice and 57\% for chalimus stages. After the treatment, only ten adult males with fully developed genital segments (mature) were recovered on the fish analysed $(n=20)$, for the rest of the adult males $(n=10)$ and all the adult females $(n=8)$ the genital segments were not fully developed ("young adult lice" following the terminology by González \& Carvajal [5]).

\section{Screening of $C$. rogercresseyi AChE1a for variants}

The screening and alignment of the full cDNA coding sequence encoding for $C$. rogercresseyi AChE1a in five sensitive (immobilized at $0.4 \mathrm{ppb}$ or below), three with reduced sensitivity (unaffected at $1.2 \mathrm{ppb}$ ) and two 
Table 1 Immobilization percentage (\%) of Caligus rogercresseyi exposed to azamethiphos per farm using bioassays

\begin{tabular}{llll}
\hline Farm code & $\begin{array}{l}\text { Aza dose } \\
(\mathrm{ppb})\end{array}$ & $\begin{array}{l}\text { Male } \\
\text { immobilization \% }(n)\end{array}$ & $\begin{array}{l}\text { Female } \\
\text { immobilization \% }(n)\end{array}$ \\
\hline 1 & 0 & $0(6)$ & $0(13)$ \\
0.2 & $14(7)$ & $0(12)$ \\
& 0.4 & $17(6)$ & $42(12)$ \\
1.2 & $100(6)$ & $91(11)$ \\
& 2.0 & $100(8)$ & $100(10)$ \\
2: Bioassay 1 & 0 & $12.5(8)$ & $0(22)$ \\
& 0.2 & $0(11)$ & $0(19)$ \\
& 0.4 & $0(11)$ & $0(20)$ \\
& 0.8 & $0(8)$ & $0(22)$ \\
& 1.2 & $42(12)$ & $37(19)$ \\
& 2 & $100(10)$ & $100(21)$ \\
2: Bioassay 2 & 0 & $0(19)$ & $0(20)$ \\
& 0.4 & $0(21)$ & $0(16)$ \\
2.0 & $93(15)$ & $93(15)$
\end{tabular}

Abbreviations: Aza azamethiphos, $n$, sample size for each louse sex

resistant (unaffected at $2 \mathrm{ppb}$ ) adult $C$. rogercresseyi, revealed several synonymous and two non-synonymous changes. Additional file 5 shows the alignment of the cDNA sequences in the ten $C$. rogercresseyi individuals. One of the two non-synonymous changes found in ace1a was thymine to guanine at position 952 (T952G). This change led to an amino acid change: phenylalanine (F) to valine $(\mathrm{V})$ at codon 318 (C. rogercresseyi AChE1a numbering). Among the ten $C$. rogercresseyi individuals analyzed, the F318V change was found in the two lice unaffected at $2 \mathrm{ppb}$ (resistant) and in one louse immobilized at $0.4 \mathrm{ppb}$ (see Table 2 for details). These three lice carrying the change were heterozygous for the variant (F/V318). The two adult lice unaffected at $2 \mathrm{ppb}$ (resistant) did not have fully developed genital segments ("young adults").

To investigate the importance of the F/V318 variant, the sequence of the $C$. rogercresseyi AChE1a protein was aligned with $19 \mathrm{AChE}$ amino acid sequences from other species (Fig. 2). The alignment revealed that F318 in C. rogercresseyi is homologous to F290 in the T. californica AChE. This amino acid is located in the acyl pocket of the protein, neighboring the catalytic center in the active-site gorge. It is a highly conserved residue among the species as evident from the MSA of AChEs from several species (Fig. 2).

The other non-synonymous change found in C. rogercresseyi acela, guanine to adenine at position 82 (G82A), led to the amino acid change valine to isoleucine at position 28 (V28I). This change was found in only one sensitive male louse and it is situated close to the $\mathrm{N}$-terminus of the protein. Valine and isoleucine are similar amino acids with similar physical properties. In addition, when aligning L. salmonis AChE1a, AChE1b and C. rogercresseyi AChE1a sequences, both L. salmonis AChEs has isoleucine at that same position (data not shown).

The analysis of the AChE1a fragment in the lice collected before and after the azamethiphos field treatment showed that every young adult female recovered after treatment carried the F/V318 variant. Table 3 shows the percentage of lice carrying the F/V318 variant in the preand post-treatment sampling, separate for sex and maturation level of the genital segment. All lice carrying the change were heterozygous for the variant (F/V318).

3D modelling of $C$. rogercresseyi AChEs and docking analysis The 3D structure of C. rogercresseyi $\mathrm{AChE1a}$ and $\mathrm{AChE} 1 \mathrm{~b}$ was modeled using the SWISS MODEL software. An

Table 2 Presence/absence of the FN318 variant in Caligus rogercresseyi AChE1a selected for azamethiphos using bioassays (10 lice)

\begin{tabular}{|c|c|c|c|c|}
\hline Aza dose (ppb) & Bioassay output & Louse sex & Farm & AChE1a variant \\
\hline 0.2 & Immobilized & Male & 1 & Homozygous F/F \\
\hline 0.4 & Immobilized & Male & 1 & Homozygous F/F \\
\hline 0.4 & Immobilized & Female & 1 & Homozygous F/F \\
\hline 0.4 & Immobilized & Female & 1 & Heterozygous F/V \\
\hline 0.4 & Immobilized & Female & 1 & Homozygous F/F \\
\hline 1.2 & Unaffected & Female & 2 & Homozygous F/F \\
\hline 1.2 & Unaffected & Female & 2 & Homozygous F/F \\
\hline 1.2 & Unaffected & Female & 2 & Homozygous F/F \\
\hline 2.0 & Unaffected & Female (young ${ }^{a}$ ) & 2 & Heterozygous F/V \\
\hline 2.0 & Unaffected & Male (young ${ }^{\mathrm{a}}$ ) & 2 & Heterozygous F/V \\
\hline
\end{tabular}

${ }^{\mathrm{a}}$ Young adult: adult lice without fully developed genital segments [5]

Lice immobilized at 0.2 and $0.4 \mathrm{ppb}$ were considered sensitive, lice unaffected at $1.2 \mathrm{ppb}$ were considered with reduced sensitivity and lice unaffected at 2 ppb, resistant

Abbreviation: Aza azamethiphos

Lice carrying the variant are highlighted in bold 
initial template search revealed several possible templates. The 3D model giving high QMEAN and GMQE scores in the template search for $C$. rogercresseyi AChE1a was the crystal structure of recombinant human AChE in the apo state (PDB code 4ey4.1) (Table 4). Native D. melanogaster AChE (PDB code 1qo9.1) gave lower GMQE and QMEAN values than human AChE as template (Table 4). Drosophila melanogaster $\mathrm{AChE}$ is the only arthropod AChE which crystalline 3D structure has been solved to date [57]. Since C. rogercresseyi is also an arthropod, $D$. melanogaster $\mathrm{AChE}$ is expected to be a relatively good template for predicting the 3D structure of $C$. rogercresseyi AChE1a. However, they belong to different AChE types, D. melanogaster AChE belonging to type 2 and C. rogercresseyi AChE to type 1 (see Fig. 4), which could explain the low GMQE and QMEAN values. The RSM values $(\mathrm{C} \alpha)$ for recombinant human $\mathrm{AChE}$, the native $D$. melanogaster AChE and the predicted structure of $C$. rogercresseyi AChE1a (F318 and V318 variants) are shown in Table 4. Since the model quality indicators and fit values were better for human $\mathrm{AChE}$ as template for the two C. rogercresseyi AChE1a variants than D. melanogaster $\mathrm{AChE}$, human AChE 3D structure was chosen to predict both variants of C. rogercresseyi AChE1a. For C. rogercresseyi $\mathrm{AChE1b}$, the crystal structure of fully glycosylated human butyrylcholinesterase (BChE) was chosen (PDB code 4aqd.1) (see Table 4 for GMQE, QMEAN and RSM values). Additional files 6, 7 and 8 contain the PDB files of the predicted C. rogercresseyi AChE1a (F318 and V318 variants) and $\mathrm{AChE} 1 \mathrm{~b}$ 3D structures.

The 3D modeling of C. rogercresseyi AChE1a predicted a gorge containing the ten amino acids important for the function of the protein. These amino acids were predicted in a similar position and orientation as the corresponding amino acids in the $D$. melanogaster and $H$. sapiens AChE templates. The predicted gorge of C. rogercresseyi AChE1a was about $16 \AA$ deep and $15 \AA$

Table 3 Percentage (\%) of Caligus rogercresseyi carrying the FN318 variant sampled before and after the azamethiphos field treatment in Farm 3

\begin{tabular}{cll}
\hline & Before treatment & After treatment \\
\hline Adult males & $8.3(1 / 12)$ & $21.1(4 / 19)$ \\
Young $^{\text {a }}$ males & Not sampled & $30(3 / 10)$ \\
Mature $^{\text {a }}$ males & $8.3(1 / 12)$ & $11.1\left(1 / 9^{\mathrm{b}}\right)$ \\
Adult females $_{\text {Young }}^{\text {a females }}$ & $50(9 / 18)$ & $100(8 / 8)$ \\
Mature $^{\text {a }}$ females & Not sampled & $100(8 / 8)$ \\
\hline
\end{tabular}

${ }^{a}$ Young adults: adult lice with the genital segment not fully developed. Mature adults: adult lice with fully developed genital segment [5]

${ }^{\mathrm{b}}$ Ten mature males recovered in total, but no sequence information from one male due to poor RNA quality

The number of lice harboring the variant out of the total number of lice per group is shown in parentheses at its widest point, close to the bottom. The entire active-site gorge had a volume similar to the gorge of the human AChE. The C. rogercresseyi AChE1a 3D model revealed that the change from phenylalanine to valine at position 318 resulted in a wider active-site gorge (Figs. 5 and 6): the gorge of the V318 variant is $8 \%$ bigger than the gorge of the F318 variant. Phenylalanine and valine have similar physical properties: both are nonpolar, hydrophobic and have neutral charge, but valine lacks the bulky aromatic ring of phenylalanine. The docking analysis predicted that acetylcholine binds in the active-site gorge of the F318 variant of C. rogercresseyi AChE1a in the proper place and orientation [56]. Acetylcholine was also predicted to bind to the active site of the V318 AChE1a variant. The organophosphates azamethiphos and dichlorvos were both predicted to bind at the mouth of the active-site gorge in the F318 variant. For azamethiphos in the V318 variant, the binding was predicted at both the mouth and the bottom of the gorge, and for dichlorvos, only at the mouth of the gorge.

The C. rogercresseyi AChE1b 3D model revealed a main gorge in the center of the protein, as in other AChEs and BChEs. The gorge is about $15 \AA$ deep and $15 \AA$ at its widest point, and its entire volume is $33 \%$ bigger than the gorge in C. rogercresseyi AChE1a F318 variant. Caligus rogercresseyi AChE1b active-site gorge is $39 \%$ smaller than human BChE. As stated above, the alignment of AChE1b and AChE1a sequences showed that some of the ten important residues that characterize a typical $\mathrm{AChE}$ are substituted by other amino acids in C. rogercresseyi AChE1b (three residues of the catalytic triad and two residues of the acyl pocket) (Fig. 2). The alignment of AChE1b and AChE1a in the 3D analysis showed that, similar to AChE1a, the substituted amino acids in AChE1b where also predicted at the bottom of the AChE1b gorge (Additional files 6 and 8). Docking analysis for AChE1b predicted that acetylcholine and azamethiphos bind inside the gorge. For dichlorvos, the analysis predicted the binding at the mouth of the gorge.

\section{Discussion}

Decreased sensitivity of sea lice for various anti-lice chemicals has become a major issue worldwide, with Chile being no exception. Pyrethroids and emamectin benzoate have been the major chemicals used aginst $C$. rogercresseyi in Chile. However, the development of resistance over the years, attributed to their overuse, has severely affected the fish farm industry [7, 9-12]. Because of this increased resistance, the organophosphate azamethiphos was introduced in 2013 in order to improve delousing treatment efficacies in the farms. Although azamethiphos treatments provided a good delousing effect during 2013-2014 in the four farms studied by Agusti et al. [7] (above 90\% 
Table 4 3D modelling of Caligus rogercresseyi AChE1a (F318 and V318 variants) and AChE1b

\begin{tabular}{|c|c|c|c|c|c|}
\hline \multirow[t]{2}{*}{ Template } & \multicolumn{2}{|l|}{ Cr AChE1a (F318) } & \multicolumn{2}{|l|}{ Cr AChE1a (V318) } & \multirow{2}{*}{$\begin{array}{l}\text { Cr AChE1b } \\
\text { Hs BChE (4aqd.1 }\end{array}$} \\
\hline & Hs AChE (4ey4.1) & Dm AChE (1qo9.1) & Hs AChE (4ey4.1) & Dm AChE (1qo9.1) & \\
\hline GMQE & 0.77 & 0.74 & 0.77 & 0.74 & 0.74 \\
\hline QMEAN & -1.66 & -3.91 & -1.66 & -3.91 & -2.87 \\
\hline RSM wp (Å) & 0.28 & 0.35 & 0.28 & 0.39 & 0.26 \\
\hline RSM 10 aa $(\AA ̊)$ & 0.13 & 0.34 & 0.13 & 0.23 & 0.25 \\
\hline
\end{tabular}

Abbreviations: Cr Caligus rogercresseyi, Hs AChE recombinant human AChE in the apo state (PDB codes are shown in parentheses), Dm AChE native Drosophila melanogaster $\mathrm{AChE}, \mathrm{Hs} B C h E$ fully glycosylated human butyrylcholinesterase, QMEAN and GMQE scores for the model quality, RMS (in $\AA$ ) fit between template and model (wp: whole protein; 10 aa: ten important amino acids)

treatment efficacy for adult lice), a loss of sensitivity was reported using bioassays. Unfortunately, no molecular marker is yet available to identify resistance in C. rogercresseyi due to the lack of knowledge on the molecular mechanisms involved in resistance towards the chemicals used. In the present study, we investigated a possible molecular mechanism of azamethiphos sensitivity loss in C. rogercresseyi.

In order to elucidate the molecular mechanisms for resistance, it is important to identify and characterize the target proteins. As per the existing literature, AChEs are the target proteins for organophosphates in arthropods (reviewed in $[24,25,28])$. In the present study, we identified two AChEs in C. rogercresseyi, AChE1a and 1b. In C. rogercresseyi $\mathrm{AChE1a}$, the in silico analysis identified it as an $\mathrm{AChE}$ with transmembrane region, and the alignment with AChEs from other species showed that it has the characteristic features of a main synaptic AChE [56]. The AChE1a 3D model positioned the ten important amino acids for the function of the protein at the bottom of the gorge, and the docking analysis predicted acetylcholine to bind at the proper site and orientation in the active-site gorge [56]. The phylogenetic analysis revealed that AChE1a clustered together with the AChE type 1 occurring in several arthropods. These results strongly indicate that AChE1a codes for the the main synaptic AChE. In silico analysis of AChE1b identified it as an AChE without transmembrane region, predicting the protein to be soluble. The phylogenetic analysis clustered AChE1b together with other invertebrate $\mathrm{AChEs}$ belonging to type 1 . However, AChE1b lacks some of the important amino acids for the function of a typical AChE. According to the

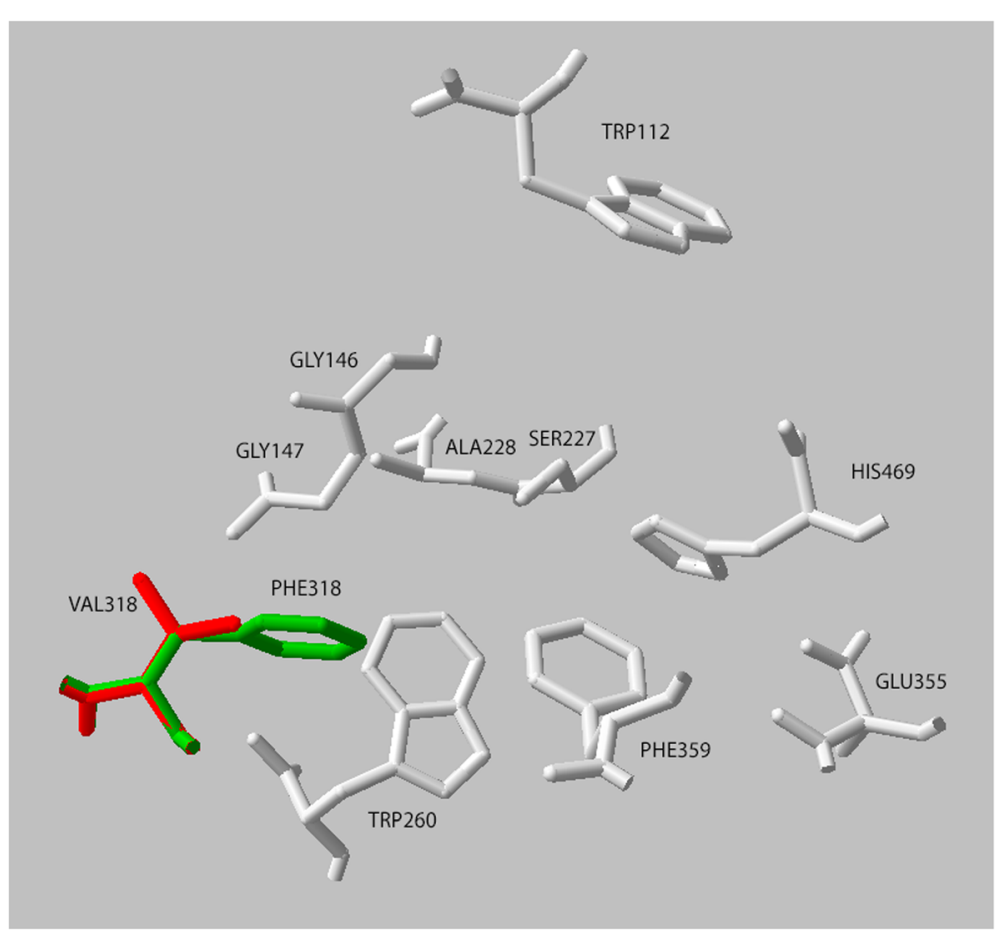

Fig. 5 Overlay of the predicted 3D positioning of the ten functionally important amino acids in both Caligus rogercresseyi AChE1a variants. Numbering corresponds to Caligus rogercresseyi AChE1a. The phenylalanine at position 318 (F290, Torpedo californica numbering) is displayed in green, while the valine at that same position is in red 


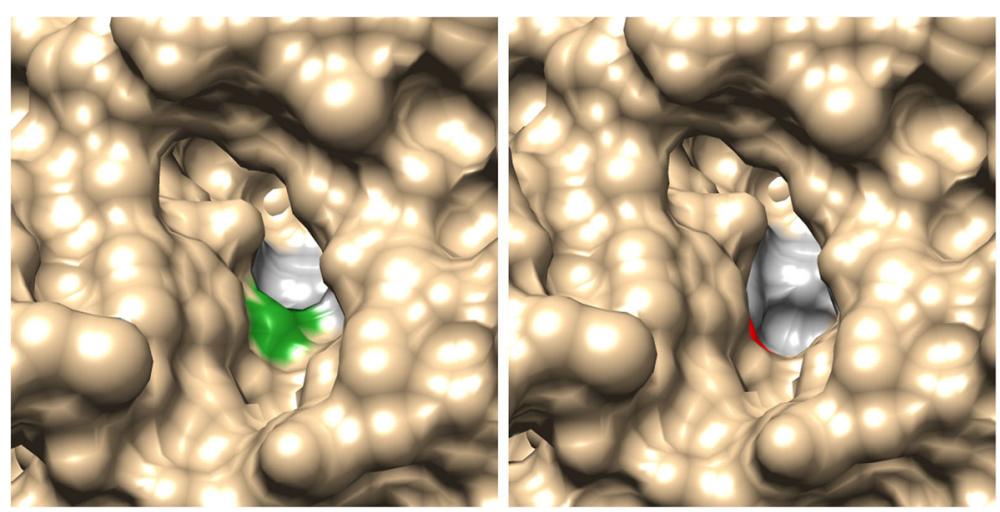

Fig. 6 Surface of the predicted 3D structure of Caligus rogercresseyi AChE1a F318 variant (left panel, displayed on green) and V318 (right panel, displayed on red). View from the mouth of the active-site gorge of the protein

docking analysis, C. rogercresseyi AChE1b could bind acetylcholine, azamethiphos and dichlorvos at different locations of the gorge. Enzymatic assays are necessary in order to properly identify the function of $C$. rogercresseyi AChE1b, but a scavenger function is possible, as is the case for vertebrate butyrylcholinesterase and for some AChE soluble forms occuring in invertebrates. In this respect, Kim et al. [58] described two AChEs in the honeybee, a membrane-anchored enzyme with a strong AChE activity and a soluble form with very low AChE activity. The authors showed that the inhibition of the main synaptic enzyme was reduced when inhibitors were pre-incubated with the soluble form. In the pinewood nematode, Kang et al. [59] also identified a soluble AChE form that conferred protection against anti-cholinesterase compounds.

Similar to C. rogercresseyi, two AChEs have been described in the other sea lice affecting farmed salmonids, L. salmonis [30]. These proteins were similar to each other and both grouped together with other invertebrate AChEs type 1 . They were named type $1 \mathrm{a}$ and $1 \mathrm{~b}$. AChE1a was predicted to be the main synaptic AChE in L. salmonis. AChE1b has also the typical AChE signature and the important amino acids for the function of an AChE. It was predicted to be a soluble protein and was suggested to be a functionally active AChE.

As per the existing literature, decreased sensitivity towards organophosphates and carbamates is mostly associated with mutations in $\mathrm{AChE}$ genes in various arthropods (reviewed in $[25,28])$. Among these, point mutations are the most commonly found. The majority of these missense mutations have been found in or around the active gorge of the enzyme, making it a hot spot for mutations. In order to correlate mutations or variants in $\mathrm{AChE}$ genes with organophosphate resistance, it is essential to properly characterize the sensitivity to the chemical of every single parasite used in the analysis. Bioassays designed for selecting individual lice are a reliable method to accomplish this [21, 22]. In our study, lice immobilized at $0.4 \mathrm{ppb}$ or below were considered sensitive and lice unaffected at $2 \mathrm{ppb}$, resistant [7, 19, 22, 23]. At the louse population level, the bioassay performed in Farm 1 showed a louse sensitivity $\left(\mathrm{EC}_{50} \sim 0.5 \mathrm{ppb}\right)$ close to the C. rogercresseyi putative naïve level $(\sim 0.15 \mathrm{ppb}$, see [7]). On the other hand, lice from Farm 2 showed reduced sensitivity towards azamethiphos $\left(\mathrm{EC}_{50} 1.2 \mathrm{ppb}\right.$ and no lice immobilized at $0.8 \mathrm{ppb}$ or below). For reference, $L$. salmonis resistant strains have $\mathrm{EC}_{50}$ values between 2.1 and $3.7 \mathrm{ppb}$ (24 h bioassay) [19, 21].

The main synaptic $C$. rogercresseyi AChE, AChE1a, was screened for variants in both azamethiphos-sensitive and -resistant lice in the present study. One non-synonymous change in the coding $\mathrm{CDNA}$ region, thymine to guanine at position 952, was found in the two C. rogercresseyi individuals surviving the highest azamethiphos concentration in the bioassay. This nucleotide change led to the amino acid change F318V (corresponding to F290 in T. californica AChE). F290 (T. californica AChE numbering) is a component part of the acyl pocket of $\mathrm{AChE}$, which is responsible for the ligand specificity, and it is highly conserved in both AChE1 and AChE2 from various species, as shown in Fig. 2. F318 (C. rogercresseyi AChE1a numbering) is therefore an important residue, most likely playing an important role in the AChE function. Furthermore, F290 ( $T$. californica AChE numbering) has been observed as one of the hot spots for mutations in $\mathrm{AChE}$, conferring different levels of resistance towards organophosphates and carbamates in various arthropods (reviewed in $[25,26,28]$ ). The most common changes reported at that position are F to V, F to L (leucine) or F to Y (tyrosine). Several examples illustrate the association between the F290V change and organophosphate/carbamate resistant in arthropods. The mutation F416V (F290V, T. californica numbering) was found in organophosphate-resistant mosquitoes Culex pipiens from Cyprus [60]. Interestingly, when the authors introduced the F416V mutation in susceptible 
mosquitoes or in recombinant enzymes by site-directed mutagenesis, they found similar levels of resistance towards a range of organophosphates and carbamates when compared to the naturally mutated resistant mosquitoes [60, 61]. Resistant mosquitoes were homozygous for the mutation, and the activity of the mutated V416 AChE1 was similar to the activity of the susceptible wild enzyme (F416). The F416V mutation persisted for at least 16 years in the mosquito population, and it was especially protective against the organophosphate dichlorvos. Interestingly, salmonid fish infested with $C$. rogercresseyi had been treated with dichlorvos for 15 years in the past in the Chilean Los Lagos Region (1985-2000, reviewed in [8]). The dichlorvos exposure may well have selected the $C$. rogercresseyi AChE1a V318 variant in louse populations in the past, conferring some protection for azamethiphos in the present.

The same residue of the acyl binding pocket was also found mutated in other organophosphate and carbamate resistant arthropods. For example, the change F399V (homozygous) was reported in carbamate and organophosphate resistant codling moth, Cydia pomonella [62]. In carbamate resistant striped rice stemborers, Chilo suppressalis, the $\mathrm{F} 402 \mathrm{~V}$ variant was found alongside with four other mutations in AChE1 (both heterozygous and homozygous individuals were found) [63]. In the organophosphate resistant fall armyworm, Spodoptera frugiperda, Carvalho et al. [26] found three amino acid substitutions in the ace-1 gene, one of them being F290V (T. californica numbering). Both heterozygous and homozygous individuals were found for this mutation. Overexpression of several genes was also found in organophosphate resistant fall armyworms. In the organophosphate resistant diamondback moth, Plutella xylostella, Yeh et al. [64] found three amino acid substitutions in AChE1. One of them was F386F/V (only heterozygous genotype was found). Their 3D modeling showed that the F386V change enlarges the space of the acetyl binding pocket. Our C. rogercresseyi AChE1a 3D model also revealed that the change from phenylalanine to valine at position 318 results in a wider active-site gorge.

The V318 variant in C. rogercresseyi AChE1a, has the same ten important amino acids as vertebrate $\mathrm{BChE}$, the latter having valine or isoleucine (depending on the species, see Additional file 9) in position 290 (T. californica $\mathrm{AChE}$ numbering). Isoleucine and valine have similar biochemical properties (conservative variation). Vertebrate $\mathrm{BChE}$ has a wider substrate specificity than $\mathrm{AChE}$, which could also be the case for the C. rogercresseyi AChE1a V318 variant. In invertebrates, Pezzementi et al. [65] investigated the acyl pocket of the amphioxus (Branchiostoma floridae) cholinesterase type 2 (ChE2) by site-directed mutagenesis. When the 312 phenylalanine of the acyl pocket (B. floridae numbering; F290 T. californica numbering) was replaced by isoleucine, a decrease in the substrate specificity of the enzyme was observed. In addition, the F312I mutation eliminated the substrate inhibition by ACh. The docking analysis performed in the present study showed that the $\mathrm{F} 318 \mathrm{~V}$ change in C. rogercresseyi AChEla could modify the binding properties of azamethiphos to the enzyme. Enzymatic assays are though warranted in order to elucidate the activity of AChE1a V318 variant in the presence and absence of organophosphates.

Only heterozygous C. rogercresseyi individuals were found in our study. Several causes could explain the lack of homozygous lice. When the frequency of the variant in the population is low, the sample size must be large to detect homozygous individuals. The percentage of males carrying the F/V318 variant sampled before the azamethiphos field treatment in Farm 3 was very low $(8.3 \%)$ and the percentaje of females was $50 \%$, therefore the probability of finding homozygous individuals is low. The F318V change could also involve a high fitness cost for the homozygous variant. However, this change has been reported as homozygous in viable resistant individuals in other arthropods (reported earlier in the discussion). In our bioassays, one lice carrying the F/V318 variant was immobilized at a low azamethiphos concentration (Table 2). The heterozygous variant may not provide full protection against organophosphates, as shown by Kaur et al. [21, 22] in L. salmonis.

In order to investigate the association between the AChE1a F/V318 variant and azamethiphos sensitivity loss, 57 C. rogercresseyi collected before and after an azamethiphos field treatment were analysed. The analysis showed that every young adult female sampled from the fish three days after treatment carried the F/ V318 variant (Table 3 ). These results indicate that the F/ V318 variant plays an important role in protecting $C$. rogercresseyi against azamethiphos. However, other mechanisms could also be involved in the protection, since lice not carrying the F/V318 variant were unaffected at $1.2 \mathrm{ppb}$ azamethiphos in the bioassays (Table 2). Behavioral avoidance, differences in the expression of the acetylcholinesterases and/or in the genes coding for detoxifying enzymes could contribute to the lice survival to azamethiphos exposure, as observed in other arthropods (reviewed in [24, 26-28]). Mobile small lice can hide under the scales of the fish, becoming less exposed to the bath treatment (C. Agusti and S. Bravo personal observation). In Farm 3, adult males without the F/V318 variant were found after the treatment. Mature adult males have higher mobility than adult females with fully developed genital segment. The post-treatment sampling occurred three days after the azamethiphos treatment, thus the mature adult males found post-treatment could be lice arriving from other untreated cages or farms. 
In L. salmonis, azamethiphos is considered to have a poorer effect against the two chalimus stages compared to preadult and adult stages. In the present study with $C$. rogercresseyi, which has four chalimus stages, $57 \%$ efficacy was recorded for chalimus. This figure should be interpreted with caution as the first two chalimus stages are difficult to see and count correctly. Within the three days from treatment to sampling, some of the chalimus IV could have moulted to young adults. Still, if the survival of the treatment was independent of the F/V318 variant, an equal proportion of F318 and F/V318 would be expected in the surviving parasites, but instead $100 \%$ of the young females sampled after treatment had the F/V318 variant. Moreover, both young adult lice (one male and one female) unaffected at $2 \mathrm{ppb}$ azamethiphos in the bioassay, were carrying the F/V318 variant.

Caligus rogercresseyi resistance towards azamethiphos is emerging and developing in Chile. In general, several resistance mechanisms can arise while resistance is developing. Although different mechanisms could be involved in the lice protection against azamethiphos, the AChE1a F/V318 variant is most probably playing a significant role in the observed reduced sensitivity. The variant could therefore be a good candidate as molecular marker, indicating loss of sensitivity in C. rogercresseyi towards organophosphates in Chile.

\section{Conclusions}

The present study reports for the first time the identification and characterization of two AChEs in C. rogercresseyi. AChE1a is most likely the main synaptic AChE, whereas $\mathrm{AChE1b}$ could be a scavenger protein. We found one variant (F/V318) in C. rogercresseyi AChE1a that could play a role in making the parasite less sensitive to azamethiphos. This change has been reported to be conferring resistance in several other arthropods towards organophosphates and carbamates. An azamethiphos field treatment showed a selection of the F/V318 variant after treatment among young adult females, indicating an association between the presence of the variant and the survival of $C$. rogercresseyi to azamethiphos. Future screening in a larger sea lice population is warranted to validate this.

\section{Additional files}

Additional file 1: Primers used for amplifying and sequencing the Caligus rogercresseyi AChE sequences found in this study. (PDF $16 \mathrm{~kb}$ )

Additional file 2: Caligus rogercresseyi AChE sequences in Whole-Genome Shotgun: contigs. (PDF $6 \mathrm{~kb}$ )

Additional file 3: Full list of abbreviations and UniprotKB database entry names from data used in Figs. 2 and 4 and Additional file 4. (PDF 21 kb)

Additional file 4: Phylogenetic analysis including Caligus rogercresseyi AChE1a and AChE1b together with several invertebrate and vertebrate carboxylesterases, esterases and other cholinesterases. Crustacean proteins are shown in red letters. Branches in red correspond to proteins tentatively assigned to the AChE type in the present analysis. For abbrevations and UniprotKB database entry names see Additional file 3. (PDF $170 \mathrm{~kb}$ )

Additional file 5: Alignment of the full coding ace la cDNA sequence from 10 Caligus rogercresseyi. The lack of the star symbol under the sequences indicates a SNP. Every individual is represented by the two cDNA variants ( $a$ and b), according to the SNPs found. Individual louse code: resistant $(L R 1, L R 2)$, reduced sensitivity $(A L, B L, C L)$, sensitive $(S S 1$, S1, S2, S3, S4). (PDF $118 \mathrm{~kb})$

Additional file 6: PDB file of the predicted 3D structure of Caligus rogercresseyi AChE1a F318 variant. Viewer: UCSF Chimera 1.10.2. software. https://www.cgl.ucsf.edu/chimera. (PDB 342 kb)

Additional file 7: PDB file of the predicted 3D structure of Caligus rogercresseyi AChE1a V318 variant. Viewer: UCSF Chimera 1.10.2. software. https://www.cgl.ucsf.edu/chimera. (PDB 331 kb)

Additional file 8: PDB file of the predicted 3D structure of Caligus rogercresseyi AChE1b. Viewer: UCSF Chimera 1.10.2. software. https:// www.cgl.ucsf.edu/chimera. (PDB 296 kb)

Additional file 9: Alignment of six vertebrate butyrylcholinesterases (BChE), Caligus rogercresseyi AChE1a V318 variant (M) and Caligus rogercresseyi AChE1b. Figure showing a fragment of the alignment containing the amino acid corresponding to the position F290 (Torpedo californica AChE numbering) (highlighted). (PDF $165 \mathrm{~kb}$ )

\section{Abbreviations}

3D: Three-dimensional; AChE: Acetylcholinesterase enzyme;

ChE: Cholinesterase enzyme; $\mathrm{EC}_{50}$ : The concentration of a chemical immobilizing 50\% of the parasites; F318FN: The important change found in the amino acid sequence in the main synaptic Caligus rogercresseyi AChE (AChE1a); Ki: Concentration required to produce half-maximum inhibition (docking analysis); MCMC: Markov Chain Monte Carlo analysis; MSA: Multiple Sequence Alignment; ORF: Open reading frame of a gene; $\mathrm{ppb}: \mathrm{Mg} \mathrm{I}^{-1}$; QMEAN and GMQE: Global 3D model quality estimation scores; RMS: Root mean square value for carbon alpha $(\mathrm{Ca})$ used for analyzing the fit between the selected templates and the predicted AChEs structures in the 3D analysis; UTR: Untranslated region of a gene; WGS: Whole-Genome Shotgun

\section{Acknowledgements}

We thank the staff at the fish farms (veterinarians, farm managers, farm operators) of Cermaq Chile, and especially to Cristina Winkler and Ingrid Ojeda for their assistance in the louse collection and providing all necessary data. We acknowledge Felipe Kauak, Karla Sambra and Nike Ponce for their assistance in the lice collection and performing of bioassays. We also thank Melanie Andrews for the language corrections and Patricia Gozalbes-Aparicio for the map design in Fig. 1. We sincerely thank the comments and suggestions made by the anonymous referees since they substantially improved the manuscript.

\section{Funding}

During the development of the study, the first author held a post-doctoral research fellowship from the Research Council of Norway, HAVBRUK program, project number: $234060 / E 40$. This work was partially funded by the SFI-Sea Lice Research Centre, grant number 203513/030. The funding body had no role in the design of the study, collection, analysis, interpretation of data or in writing the manuscript.

\section{Availability of data and materials}

Most data generated or analyzed during this study are included in this published article and its additional files. Additional data are available from the corresponding author upon reasonable request.

\section{Authors' contributions}

CAR and TEH were involved in the planning of the study, interpretation of the results and in writing the manuscript. CAR peformed most of the louse sampling and bioassays, as well as most of the molecular analysis. MD and KK performed some molecular analyisis and contributed in writing the manuscript. $J$ and BK contributed in the molecular analysis. SB and JM assisted in the organization and performance of the lice sampling and bioassays. All authors read and approved the final manuscript. 


\section{Ethics approval and consent to participate}

The study only involved sea lice and no fish were used. All parasites were collected from fish farms with the farmers' consent, after which no further permissions were required. The study did not involve endangered or protected species.

\section{Consent for publication}

Not applicable.

\section{Competing interests}

The authors declare that they have no competing interests.

\section{Publisher's Note}

Springer Nature remains neutral with regard to jurisdictional claims in published maps and institutional affiliations.

\section{Author details}

${ }^{1}$ Faculty of Veterinary Medicine, Norwegian University of Life Sciences, Sea Lice Research Centre, Postboks 369 Sentrum, Oslo NO-0102, Norway.

${ }^{2}$ Department of Informatics, University of Bergen, Sea Lice Research Centre, Thormøhlensgate 55, N-5008 Bergen, Norway. ${ }^{3}$ Biology Department, Centre for Biomedical Research, University of Victoria, Station CSC, PO Box 1700, Victoria, BC V8W 2Y2, Canada. ${ }^{4}$ Universidad Austral de Chile, Casilla 1327, Puerto Montt, Chile. ${ }^{5}$ Cermaq Chile, Diego Portales 2000, Puerto Montt, Chile.

Received: 19 June 2018 Accepted: 14 October 2018

Published online: 30 October 2018

\section{References}

1. Bravo S. Sea lice in Chilean salmon farms. Bull Eur Assoc Fish Pathol. 2003: 23:197-200.

2. Johnson SC, Treasurer JW, Bravo S, Nagasawa K. Kabata Z. A review of the impact of parasitic copepods on marine aquaculture. Zool Stud. 2004;43: 229-43.

3. Lhorente JP, Gallardo JA, Villanueva B, Carabaño MJ, Neira R. Disease resistance in Atlantic salmon (Salmo salar): coinfection of the intracellular bacterial pathogen Piscirickettsia salmonis and the sea louse Caligus rogercresseyi. PLoS One. 2014;9:e95397.

4. González MP, Muñoz JLP, Valerio V, Vargas-Chacoff L. Effects of the ectoparasite Caligus rogercresseyi on Salmo salar blood parameters under farm conditions. Aquaculture. 2016;457:29-34.

5. González L, Carvajal J. Life cycle of Caligus rogercresseyi, (Copepoda: Caligidae) parasite of Chilean reared salmonids. Aquaculture. 2003:220:101-17.

6. Aaen SM, Helgesen KO, Bakke MJ, Kaur K, Horsberg TE. Drug resistance in sea lice: a threat to salmonid aquaculture. Trends Parasitol. 2015;31:72-81.

7. Agusti C, Bravo S, Contreras G, Bakke MJ, Helgesen KO, Winkler C, et al. Sensitivity assessment of Caligus rogercresseyi to anti-louse chemicals in relation to treatment efficacy in Chilean salmonid farms. Aquaculture. 2016; 458:195-205.

8. Bravo S, Sepulveda M, Silva MT, Costello MJ. Efficacy of deltamethrin in the control of Caligus rogercresseyi (Boxshall and Bravo) using bath treatment. Aquaculture. 2014:432:175-80

9. Bravo S, Sevatdal S, Horsberg TE. Sensitivity assessment of Caligus rogercresseyi to emamectin benzoate in Chile. Aquaculture. 2008:282:7-12.

10. Bravo S, Sevatdal S, Horsberg TE. Sensitivity assessment in the progeny of Caligus rogercresseyi to emamectin benzoate. Bull Eur Assoc Fish Pathol. 2010;30:92-8.

11. Bravo S, Silva MT, Monti G. Efficacy of emamectin benzoate in the control of Caligus rogercresseyi on farmed Atlantic salmon (Salmo salar L.) in Chile from 2006 to 2007. Aquaculture. 2012;364-365:61-6.

12. Helgesen KO, Bravo S, Sevatdal S, Mendoza J, Horsberg TE. Deltamethrin resistance in the sea louse Caligus rogercresseyi (Boxhall and Bravo) in Chile: bioassay results and usage data for antiparasitic agents with references to Norwegian conditions. J Fish Dis. 2014;37:877-90.

13. Sernapesca. Informe Sanitario de Salmonicultura en Centros Marinos. 2016 http://www.sernapesca.cl. Accessed 19 Sept 2017.

14. Denholm I, Devine GJ, Horsberg TE, Sevatdal S, Fallang A, Nolan DV, et al. Analysis and management of resistance to chemotherapeutants in salmon lice Lepeophtheirus salmonis (Krøyer) (Copepoda: Caligidae). Pest Manag Sci. 2002;58:528-36.
15. Jones MW, Sommerville C, Wootten R. Reduced sensitivity of the salmon louse, Lepeophtheirus salmonis, to the organophosphate dichlorvos. J Fish Dis. 1992;15:197-202.

16. Roth M, Richards RH, Dobson DP, Rae GH. Field trials on the efficacy of the organophosphorus compound azamethiphos for the control of sea lice (Copepoda: Caligidae) infestations of farmed Atlantic salmon (Salmo salar). Aquaculture. 1996;140:217-39.

17. Tully O, McFadden Y. Variation in sensitivity of sea lice Lepeophtheirus salmonis (Krøyer) to dichlorvos on Irish salmon farms in 1991-92. Aquac Res. 2000:31:849-54.

18. Marín SL, Ibarra R, Medina MH, Jansen PA. Sensitivity of Caligus rogercresseyi (Boxshall and Bravo 2000) to pyrethroids and azamethiphos measured using bioassay tests - a large scale spatial study. Prev Vet Med. 2015;122:33-41.

19. Helgesen $\mathrm{KO}$, Horsberg TE. Single-dose field bioassay for sensitivity testing in sea lice, Lepeophtheirus salmonis: development of a rapid diagnostic tool. J Fish Dis. 2013;36:261-72.

20. Robertson $\mathrm{L}$, Preisler HK, Ng SS, Hickle LA, Gelernter WD. Natural variation: a complicating factor in bioassays with chemical and microbial pesticides. J Econ Entomol. 1995;88:1-10.

21. Kaur K, Helgesen KO, Bakke MJ, Horsberg TE. Mechanism behind resistance against the organophosphate azamethiphos in salmon lice (Lepeophtheirus salmonis). PLoS One. 2015b;10:e0124220.

22. Kaur K, Jansen PA, Aspehaug VT, Horsberg TE. Phe362Tyr in AChE: A major factor responsible for azamethiphos resistance in Lepeophtheirus salmonis in Norway. PLoS One. 2016:11:e0149264.

23. Jansen PA, Grøntvedt RN, Tarpai A, Helgesen KO, Horsberg TE. Surveillance of the sensitivity towards antiparasitic bath-treatments in the salmon louse (Lepeophtheirus salmonis). PLoS One. 2016;11:e0149006.

24. Siegfried BD, Scharf ME. Mechanisms of organophosphate resistance in insects. In: Ishaaya I, editor. Biochemical sites of insecticide action and resistance. Heidelberg-Berlin: Springer; 2001. p. 269-91.

25. Hotelier $T$, Nègre $V$, Marchot $P$, Chatonnet $A$. Insecticide resistance through mutations in cholinesterases or carboxylesterases: data mining in the ESTHER database. J Pestic Sci. 2010:35:315-20.

26. Carvalho RA, Omoto C, Field LM, Williamson MS, Bass C. Investigating the molecular mechanisms of organophosphate and pyrethroid resistance in the fall armyworm Spodoptera frugiperda. PLoS One. 2013;8:e62268.

27. Nansen C, Baissac O, Nansen M, Powis K, Baker G. Behavioral avoidance will physiological insecticide resistance level of insect strains affect their oviposition and movement responses? PLoS One. 2016;11:e0149994.

28. Guo D, Luo J, Zhou Y, Xiao H, He K, Yin C, et al. ACE: an efficient and sensitive tool to detect insecticide resistance-associated mutations in insect acetylcholinesterase from RNA-Seq data. BMC Bioinformatics. 2017;18:330.

29. Toutant JP. Insect acetylcholinesterase: catalytic properties, tissue distribution and molecular forms. Prog Neurobiol. 1989:32:423-46.

30. Kaur K, Bakke MJ, Nilsen F, Horsberg TE. Identification and molecular characterization of two acetylcholinesterases from the salmon louse, Lepeophtheirus salmonis. PLoS One. 2015a;10:e0125362.

31. Leong JS, Bravo S, von Schalburg KR, Koop BF. Caligus rogercresseyi transcriptome shotgun assembly (TSA) (11.09.2014). GenBank number: GAZX00000000.1. Centre for Biomedical Research, University of Victoria, Canada. 2014. https://www.ncbi.nlm.nih.gov/nuccore/604768531. Accessed 19 Sept 2017

32. NCBI, National Center for Biotechnology Information. Basic Local Alignment Search Tool. http://blast.ncbi.nlm.nih.gov. Accessed 19 Sept 2017.

33. Leong JS, Rondeau EB, Koop BF. Caligus rogercresseyi whole-genome shotgun contigs (WGS). GenBank numbers: LBBV00000000.1 (female; 08.05. 2015) and LBBU00000000.1 (male; 07.05.2015). Centre for Biomedical Research, University of Victoria, Canada. 2015. https://www.ncbi.nlm.nih.gov/ nuccore/LBBV00000000.1; https://www.ncbi.nlm.nih.gov/nuccore/819231677. Accessed 19 Sept 2017.

34. Sievers F, Wilm A, Dineen D, Gibson TJ, Karplus K, Li W, et al. Fast, scalable generation of high-quality protein multiple sequence alignments using Clustal Omega. Mol Syst Biol. 2011;7:539.

35. Hirokawa T, Boon-Chieng S, Mitaku S. SOSUl: classification and secondary structure prediction system for membrane proteins. Bioinformatics. 1998;14:378-9.

36. SOSUI system. http://harrier.nagahama-i-bio.ac.jp/sosui/sosui_submit.html (Accessed 19 Sept 2017).

37. Sonnhammer EL, von Heijne G, Krogh A. A hidden Markov model for predicting transmembrane helices in protein sequences. Proc Int Conf Intell Syst Mol Biol. 1998;6:175-82. 
38. TMHMM Server v. 2.0. http://www.cbs.dtu.dk/services/TMHMM/ (Accessed 19 Sept 2017).

39. Krogh A, Larsson B, von Heijne G, Sonnhammer ELL. Predicting transmembrane protein topology with a hidden Markov Model: application to complete genomes. J Mol Biol. 2001;305:567-80.

40. Cserzo M, Wallin E, Simon I, Von Heijne G, Elofsson A. Prediction of transmembrane alpha-helices in procariotic membrane proteins: the Dense Alignment Surface method. Prot Eng. 1997;10:673-6.

41. "DAS" - Transmembrane Prediction server. https://tmdas.bioinfo.se/DAS/ (Accessed 19 Sept 2017).

42. Edgar RC. MUSCLE: Multiple sequence alignment with high accuracy and high throughput. Nucl Acids Res. 2004;32:1792-7.

43. Clamp M, Cuff J, Searle SM, Barton GJ. The Jalview Java Alignment Editor. Bioinformatics. 2004;20:426-7.

44. Huelsenbeck JP, Ronquist F. MRBAYES: Bayesian inference of phylogenetic trees. Bioinformatics. 2001;17:754-5.

45. Ronquist F, Huelsenbeck JP. MrBayes 3: Bayesian phylogenetic inference under mixed models. Bioinformatics. 2003;19:1572-4.

46. Abascal F, Zardoya R, Posada D. ProtTest: Selection of best-fit models of protein evolution. Bioinformatics. 2005;21:2104-5.

47. Arnold K, Bordoli L, Kopp J, Schwede T. The SWISS-MODEL workspace: a web-based environment for protein structure homology modelling. Bioinformatics. 2006;22:195-201.

48. Bordoli L, Kiefer F, Arnold K, Benkert P, Battey J, Schwede T. Protein structure homology modelling using SWISS-MODEL workspace. Nat Protoc. 2009:4:1-13.

49. Biasini M, Bienert S, Waterhouse A, Arnold K, Studer G, Schmidt T, Kiefer $F$, et al. SWISS-MODEL: modelling protein tertiary and quaternary structure using evolutionary information. Nucleic Acids Res. 2014;42:W252-8.

50. Guex N, Peitsch MC, Schwede T. Automated comparative protein structure modeling with SWISS-MODEL and Swiss-PdbViewer: A historical perspective. Electrophoresis. 2009;30:S162-73.

51. Pettersen EF, Goddard TD, Huang CC, Couch GS, Greenblatt DM, Meng EC, et al. UCSF Chimera--a visualization system for exploratory research and analysis. J Comput Chem. 2004;25:1605-12.

52. Dundas J, Ouyang Z, Tseng J, Binkowski A, Turpaz Y, Liang J. CASTp: computed atlas of surface topography of proteins with structural and topographical mapping of functionally annotated residues. Nucl Acids Res. 2006;34:W116-8

53. CASTp server. http://sts.bioe.uic.edu/castp (Accessed 19 Sept 2017).

54. Bikadi Z, Hazai E. Application of the PM6 semi-empirical method to modeling proteins enhances docking accuracy of AutoDock. J Cheminform. 2009;1:15.

55. Dockingserver. http://www.dockingserver.com/web (Accessed 19 Sept 2017).

56. Dvir H, Silman I, Harel M, Rosenberry TL, Sussman JL. Acetylcholinesterase: from 3D structure to function. Chem Biol Interact. 2010;187:10-22.

57. Harel M, Kryger G, Rosenberry TL, Mallender WD, Lewis T, Fletcher RJ, et al. Three-dimensional structures of Drosophila melanogaster acetylcholinesterase and of its complexes with two potent inhibitors. Protein Sci. 2000;9:1063-72.

58. Kim YH, Cha DJ, Jung JW, Kwon HW, Lee SH. Molecular and kinetic properties of two acetylcholinesterases from the western honey bee, Apis mellifera. PLoS One. 2012;7:e48838.

59. Kang JS, Lee DW, Koh YH, Lee SH. A soluble acetylcholinesterase provides chemical defense against xenobiotics in the pinewood nematode. PLoS One. 2011;6:e19063.

60. Alout H, Berthomieu A, Hadjivassilis A, Weill M. A new amino-acid substitution in acetylcholinesterase 1 confers insecticide resistance to Culex pipiens mosquitoes from Cyprus. Insect Biochem Molec. 2007;37:41-7

61. Alout $\mathrm{H}$, Weill M. Amino-acid substitutions in acetylcholinesterase 1 involved in insecticide resistance in mosquitoes. Chem Biol Interact. 2008; 175:138-41.

62. Cassanelli S, Reyes M, Rault M, Manicardi GC, Sauphanor B. Acetylcholinesterase mutation in an insecticide-resistant population of the codling moth Cydia pomonella (L.). Insect Biochem Molec. 2006;36:642-53.

63. Chang C, Cheng X, Huang XY, Dai SM. Amino acid substitutions of acetylcholinesterase associated with carbofuran resistance in Chilo suppressalis. Pest Manag Sci. 2014;70:1930-5.
64. Yeh S, Lin C, Chang C, Feng H, Dai S. Amino acid substitutions and intron polymorphism of acetylcholinesterase 1 associated with mevinphos resistance in diamondback moth, Plutella xylostella (L.). Pestic Biochem Phys. 2014;112:7-12.

65. Pezzementi L, Johnson K, Tsigelny I, Cotney J, Manning E, Barker A, et al. Amino acids defining the acyl pocket of an invertebrate cholinesterase. Comp Biochem Physiol. 2003;136:813-32.

\section{Ready to submit your research? Choose BMC and benefit from:}

- fast, convenient online submission

- thorough peer review by experienced researchers in your field

- rapid publication on acceptance

- support for research data, including large and complex data types

- gold Open Access which fosters wider collaboration and increased citations

- maximum visibility for your research: over $100 \mathrm{M}$ website views per year

At BMC, research is always in progress.

Learn more biomedcentral.com/submissions 